\title{
Mujeres en la educación: desigualdades sociales más allá del género*
}

[Artículos]

Claudia Bibiana Ruiz ${ }^{* *}$

Recibido: 27/10/2020

Aprobado: 24/11/2020

Citar como:

Ruíz, C. (2021). Mujeres en la educación: desigualdades sociales más allá del género. Análisis, 53(98). https://doi.org/10.15332/21459169.6237

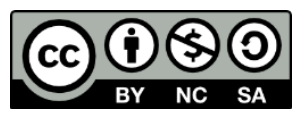

\section{Resumen}

El presente es un ensayo teórico-reflexivo que pretende hacer una aproximación a la manera en que el rol de la mujer ha estado sesgado por representaciones sociales, heredadas por generaciones. En estas representaciones, los hombres están más estrechamente asociados con el trabajo y el desarrollo profesional, mientras las féminas se vinculan principalmente con las labores de la casa y de la familia. Uno de los epicentros de esta problemática, que se suma a lo anterior, es la educación. Con todo, en América Latina, y en concreto en Colombia, las desigualdades sociales son muy profundas y reflejan una brecha de género, reproducida permanentemente, que podría ser rastreada en

\footnotetext{
* Artículo de reflexión.

** Doctoranda en Ciencias Humanas y Sociales de la Universidad Nacional de Colombia. Profesora-investigadora en la Universidad Santo Tomás (Sede Villavicencio) y la Universidad Sergio Arboleda (Barranquilla). Correo electrónico:

claudiabruiz@usantotomas.edu.co; ORCID: https://orcid.org/0000-0002-2587-9088
} 
términos de colonización e interseccionalidad. Este documento abordará tres momentos clave. En primer lugar, se sitúa a las mujeres en la educación; en segundo lugar, se observan, desde una mirada general, los desafíos en términos de equidad de género para la educación superior (ES) a partir de la incorporación de las Tecnologías de la Información y de la Comunicación (TIC), y, finalmente, se expone una perspectiva metodológica en clave interseccional, que logre dialogar entre los momentos enunciados. Se pretende hacer un primer abordaje a este concepto desde la perspectiva educativa como una oportunidad que dé cuenta de la promesa de inclusión social y equidad para las mujeres en la academia.

Palabras clave: TIC, educación superior, equidad de género, desigualdad, interseccionalidad.

\section{Women in education: social inequalities beyond gender}

\section{Abstract}

This is a theoretical-reflective essay that aims to approach the way in which the role of women has been biased by social representations, inherited by generations. In these representations, men are more closely associated with work and professional development, while women are mainly associated with housework and family. One of the epicenters of this problem, in addition to the above, is education. Overall, in Latin America, and specifically in Colombia, social inequalities are very deep and reflect a gender gap, permanently reproduced, which could be traced in terms of colonization and intersectionality. This paper will address three key moments. Firstly, it places women in education; secondly, it observes, from a general point of view, the challenges in terms of gender equity for higher education (HE) from the incorporation of the Information and 
Communication Technologies (ICT), and finally, it presents an intersectional methodological perspective, which manages to dialogue between the aforementioned moments. It is intended to make a first approach to this concept from the educational perspective as an opportunity that realizes the promise of social inclusion and equity for women in the academy.

Keywords: ICT, higher education, gender equity, inequality, intersectionality

\section{Mulheres na educação: desigualdades sociais além do gênero}

\section{Resumo}

O presente é um ensaio teórico-reflexivo que visa aproximar-se da forma como o papel das mulheres tem sido tendencioso pelas representações sociais, herdadas por gerações. Nessas representações, os homens estão mais associados ao trabalho e ao desenvolvimento profissional, enquanto as mulheres estão associadas principalmente ao trabalho doméstico e familiar. Um dos epicentros desse problema, que se soma ao dito anteriormente, é a educação. No entanto, na América Latina, e especificamente na Colômbia, as desigualdades sociais são muito profundas e refletem uma lacuna de gênero, permanentemente reproduzida, que poderia ser rastreada em termos de colonização e interseccionalidade. Este documento abordará três momentos-chave. Em primeiro lugar, situa as mulheres na educação; em segundo lugar, observa, de uma perspectiva geral, os desafios em termos de equidade de gênero para o ensino superior(ES) a partir da incorporação de Tecnologias da Informação e Comunicação (TIC), e, por fim, uma perspectiva metodológica é exposta numa área interseccional, que consegue dialogar entre os momentos enunciados. Pretende-se fazer uma primeira abordagem desse conceito do ponto 
de vista educacional como uma oportunidade que concretize a promessa de inclusão social e equidade para as mulheres na academia.

Palavras-chave: TIC, ensino superior, equidade de gênero, desigualdade, interseccionalidade.

\title{
Introducción
}

\author{
No hay barrera, cerradura ni \\ cerrojo que puedas imponer a la \\ libertad de mi mente. \\ Virginia Woolf
}

La literatura contemporánea documenta ampliamente la forma en que la dominación, desde la época de la Colonia, ha perfilado a las mujeres en oficios histórica y hereditariamente domesticados, mientras que los hombres pueden acceder a diferentes servicios y derechos como la educación, en aras de un progreso y desarrollo personal y profesional, que les permita mejorar su calidad de vida. La predominancia de los estereotipos de género ha tenido una amplia influencia respecto a los ideales que se espera sean y hagan las mujeres y, por ende, los hombres. Estos roles de género han marcado el paso de generaciones respecto al qué y cómo deben ser las personas en la sociedad.

Es importante mencionar que el concepto de estereotipo fue abordado por Smith y Mackie (1997) como una representación cognitiva que emerge y se reproduce en un grupo social determinado; son históricos y hacen parte de la estructura cultural de este. Así como señalaron Durkheim y posteriormente Moscovici (1961), el estereotipo se inserta en las representaciones sociales (RS) debido a que estas se configuran como sistemas de valores, de ideas y prácticas con doble función: 1) para darle orden y sentido a las acciones y pensamientos de los individuos, es decir, 
poder orientarse en el mundo socio-material, con el fin de dominarlo; 2) para generar la comunicación entre humanos que comparten códigos comunes y así intercambiar ideas sobre cómo nombrar y clasificar aspectos de su mundo, de su historia individual y colectiva.

Según Bourdieu (2000), como lo citó Kubissa (2017), uno de los lugares en los que la dominación masculina se manifiesta de manera más indiscutible y visible es en la unidad doméstica. En este espacio se reproducen las relaciones de fuerza materiales y simbólicas que allí se ejercen situadas en instituciones como la iglesia, la escuela o el Estado. Es común escuchar frases como "los hombres no lloran", "las mujeres no saben conducir" o "ellas son mejores en profesiones del cuidado" (Cook y Cusack, 2010, p. 1). Sin duda, en estas oraciones usuales se plantean las representaciones sobre lo deseable del comportamiento de la mujer y del hombre, lo que invisibiliza a las personas y sus necesidades.

Según Bourdieu (2002, 2014), las estructuras que organizan de forma relacionada las matrices de dominación de las sociedades, también establecen posiciones de privilegio y dominación según la clase social, la edad, la raza, el género, etc. Estas se "presentan" en el mundo social de forma objetivada y visible (por ejemplo, color de piel, rasgos faciales y corporales, vestimenta, etc.) y de forma incorporada y pegada a la piel (formas de andar, de gesticular con las manos, de sentarse, de tocar, entre otros). Así pues, se convierten en disposiciones corporales (habitus) que facilitan una forma de "violencia simbólica" difícilmente sistematizable en un formulario de demandas sociales.

En este sentido, la realidad social muta y se transforma de acuerdo con las condiciones socioculturales e históricas de una sociedad. A pesar de las luchas históricas y mundiales de movimientos feministas por disminuir la brecha ante la desigualdad en las relaciones de género en el campo 
académico y laboral, se han construido y naturalizado etiquetas o atributos que clasifican, nombran y operan como estereotipos, que permanecen de forma silenciosa como parte de la violencia cultural y estructural a la que se refiere Galtung (2000). Sin embargo, es justo decir que hay muchas cuestiones que afectan a las mujeres más allá del género, debido a que estas no pueden abordarse como una masa homogénea.

En cuanto al concepto de género, la Real Academia Española (RAE) lo define como "grupo al que pertenecen los seres humanos de cada sexo, entendido este desde un punto de vista sociocultural en lugar de exclusivamente biológico". Sin embargo, desde la mirada de las teorías feministas, este concepto es, además, un sinónimo de acciones, enfoques y programas que tienen que ver con las "mujeres", en el que género se relaciona con otros términos que también son de análisis social. Incluso, se profundiza en el motivo de las diferencias entre ellos y ellas y la manera en que se construyen, sostienen y benefician tanto a hombres como a féminas en circunstancias y situaciones dadas. Este concepto ha sido incluido en la formulación de políticas públicas y, por esta razón, la igualdad de género ha aparecido como una prioridad en instrumentos internacionales como los Objetivos de Desarrollo del Milenio (ODM) y ahora los Objetivos de Desarrollo Sostenible (ODS) aprobados por la Asamblea de las Naciones Unidas en septiembre de 2015 .

Además del género, se deben tener en cuenta otras circunstancias como la clase social, la edad, la etnia, para obtener una idea más integral de la conformación de un individuo y de sus relaciones sociales. Oakley (1972) como se citó en Unesco (2016) “observó en sus estudios realizados, la forma en que niños y niñas de la clase obrera americana aprendían antes los papeles de género que las niñas de clase media” (p. 20). Recientemente, se pudo comprender la relación entre género, 
analfabetismo y localidad geográfica gracias a que la Unesco informó que, en pleno siglo XXI, más de 700 millones de personas en el mundo son analfabetas y, de esta cifra, 493 millones son féminas que se encuentran en su mayoría en zonas rurales (Unesco, 2016).

En cuanto a la interseccionalidad ${ }^{1}$, es pertinente identificar las múltiples identidades que confluyen en una persona o colectivo y, asimismo, entender las desventajas o privilegios que se le presentan a lo largo de su vida. Por ejemplo, el acceso a oportunidades y ejercicio de derechos de una fémina afrodescendiente, adulta mayor, con una discapacidad, es distinta de una mujer blanca, joven, en una zona urbana. Si bien la naturaleza de este artículo no es hacer una revisión del término de interseccionalidad, sí es fundamental resaltar los seis principios encontrados por autores como Cooper (2015), Collins y Bilge (2016), los cuales son descritos en un apartado más adelante. Sus planteamientos permiten trazar una ruta de comprensión respecto a la interseccionalidad como una "forma para entender y analizar la complejidad del mundo, de los seres humanos y sus experiencias" (Collins y Bilge, 2016, p. 25).

$\mathrm{Al}$ abordarse el género sin la interseccionalidad como herramienta de análisis, la reproducida y naturalizada discriminación (raza, género, entre otras) e invisibilización de determinadas mujeres en diferentes áreas disciplinares o de conocimiento puede seguir influyendo en el acceso que ellas pueden tener a diferentes derechos y oportunidades (académicas y laborales) en tensiones constantes de opresión y privilegio. Según López (1992), "lo femenino ha sido devaluado de modo consistente en el sistema educativo [...] la producción cultural de la mujer se ha silenciado, trivializado o distorsionado en la construcción del saber que han hecho los

\footnotetext{
${ }^{1}$ Concepto que, en derechos humanos y género, es aplicable tanto a hombres como a mujeres.
} 
hombres" (p. 4). Un caso ilustrativo fue la ideología del mestizaje que prevaleció hasta finales del siglo XX, en el que se buscaba privilegiar la homogeneidad nacional enmarcada en una misma religión, lengua y raza. En este caso se ejemplifica que la palabra, la educación y el poder eran, en mayor medida, liderados por hombres.

En consecuencia, desde 1989, año en el que la abogada afroestadounidense Crenshaw acuñó el término de interseccionalidad, se empieza a pensar en la articulación entre los sistemas de opresión. De igual forma, se confrontó el hecho de que muchos de los problemas de justicia social, como el racismo y el sexismo, a menudo se superponen y crean múltiples niveles de injusticia social en todos los escenarios, ya sean culturales, sociales, académicos, laborales etc. En los años 90, los cuestionamientos sobre el centralismo de tal homogeneidad no se hicieron esperar. Por ende, surgió la redefinición del relato nacional, que alcanzó niveles constitucionales con el reconocimiento de la multiculturalidad y la creación de políticas públicas dirigidas a los grupos en "desventaja”. Incluso, la palabra raza quedó prohibida por concebirse como racista per sé. Por consiguiente, para entender dicho concepto, se deben analizar de manera simultánea las dimensiones raciales y de género, a partir de una perspectiva interseccional.

Con el concepto interseccional se inició una suerte de superación de la conceptualización matemática de las desigualdades sociorraciales. En este sentido, es importante resaltar que esta puede ser entendida como algo que ayuda a comprender la complejidad social, no como algo que alinea las opresiones o la dominación, sino que trata de indagar la forma en que se da la dinámica entre sexo, género, clase y raza, en unos contextos de dominación que han sido históricamente construidos. 
La relevancia de tomar en cuenta las féminas y la educación en el mundo contemporáneo mediado por las TIC radica en que la educación, como derecho fundamental, es una víctima de las diferentes desigualdades que van más allá del género. La educación, especialmente la de niñas y mujeres, es una de las inversiones más efectivas para la paz y el desarrollo sostenible (Arango y Corona, 2016). La educación también es uno de los principales espacios para promover la igualdad de género, en el que todas las personas deberían poder gozar de manera equitativa las mismas oportunidades de acceso a una formación de calidad. Lo anterior debido a que esta área del conocimiento brinda formación académica, valores y conocimientos indispensables para desarrollar las habilidades humanas y, de esta manera, poder mejorar la calidad de vida de las mujeres en lo personal, lo social y lo económico.

\section{Las mujeres y la educación}

En las últimas décadas, frases como "paz, equidad y educación" en Colombia han sido protagonistas en las propuestas de políticas públicas que esperan ser la esperanza de grupos vulnerables, personas de bajos recursos económicos, comunidades indígenas, individuos con discapacidad, afrodescendientes y, dentro de estas características, en su gran mayoría, también féminas. Aunque el Gobierno Nacional ha generado algunas iniciativas que suponen mejoran los procesos de equidad e inclusión social, la distribución desigual de la educación sigue siendo una constante.

A pesar de los cambios del sistema socioeducativo, en el que se introducían modificaciones en la enseñanza primaria y secundaria, estos últimos también se hacían extensivos a la enseñanza femenina. Sobre todo, desde el Decreto 227 de 1933 (2 de febrero), reformatorio del Decreto 1487 de 
1932 (13 de septiembre) ${ }^{2}$, en el que a la fémina se le ofrece la oportunidad y el acceso a la educación formal. Sin embargo, después de más de setenta años, la fémina sigue siendo vista como la que debe cumplir las labores de cuidado en el hogar. "Desde una edad muy temprana se socializa a los niños y las niñas para que adopten los ideales concretos de la masculinidad y la feminidad" (Marcovici, 2002, p. 4), situación que dificulta la vinculación con diferentes campos del saber para desarrollar sus habilidades científicas e intelectuales.

Según el Informe Mundial sobre la Educación de la Unesco (1991), más del $30 \%$ de las mujeres eran analfabetas. Tal es el caso de Bolivia, El Salvador y Guatemala, donde las áreas rurales son las más afectadas. Desde el análisis de datos, las cifras demuestran que existe una predominancia en que la población infantil pasa alguna vez por la escuela, siendo en su mayoría una representación femenina. Sin embargo, esto no significa que permanezcan allí. Las cifras de deserción escolar dejan entrever que, en los países de América Latina, sólo el 70 \% de los estudiantes matriculados completan la escuela primaria. Ya a nivel secundario, aunque el acceso es heterogéneo y la predominancia es femenina, las mujeres tienen preferencia por una educación media que conduzca a la universidad o a profesiones del sector servicios tales como el bachillerato o la escuela comercial. Los varones, en cambio, son mayoría en los establecimientos de educación técnica y agropecuaria.

\footnotetext{
${ }^{2}$ La segunda enseñanza en lo sucesivo comprenderá seis años, cuya mínima extensión de estudios será necesaria lo mismo para los alumnos que aspiren a ingresar a la universidad como para aquellos que quieran seguir la carrera del magisterio, o solamente adquirir el título de bachiller. Terminados los estudios de segunda enseñanza, el alumno que aspire al título de bachiller o al certificado de estudios para ingreso a la universidad, refrendado por el Gobierno, deberá someterse previamente a un examen de cultura general, en desarrollo de lo ordenado por el artículo $9 .^{\circ}$ de la Ley 56 de 1927. Este examen tendrá lugar en la capital de la República o en las capitales de los Departamentos [...].
} 
Entre otras desigualdades en América Latina, respecto a lo que ES se refieren, Chiroleu (2014) afirmó que se debe aludir a otros factores que influyen: desigualdades económicas, desigualdades étnico-raciales, desigualdades derivadas de la concentración urbana y regional de la oferta universitaria, desigualdades relativas a la permanencia y el egreso y las desigualdades de género. En esta misma línea, Martínez (2013) ilustra el caso de México, en el que las desigualdades sociales, en el marco de la ES, se asocian a conceptos como pobreza, lucha de clases, marginación, desventajas individuales y colectivas que sobrepasan la clasificación del género y, por consiguiente, donde la educación supone ser el vehículo más importante para la movilidad social (d'Addio, 2007).

De manera general, se puede detallar la forma en que los países del mundo entero buscan reducir las desigualdades heredadas del estatus socioeconómico y garantizar, al menos, un mínimo nivel de estudios, siendo la ES una forma de desarrollo, puesto que supone traerá mayor oportunidad laboral, competitividad, equidad, igualdad y flexibilidad de la economía. Sin duda, este panorama es un desafío para las Instituciones de Educación Superior (IES) que han evitado, como si fuesen piedras, las cuestiones de equidad y género en el interior de los diferentes espacios y procesos que se hacen más visibles a partir de diferentes diagnósticos e investigaciones. Tal es el caso de los hallazgos que el Boletín del Observatorio de Asuntos de Género de la Universidad Nacional de Colombia dio a conocer por primera vez en el año 2017. En este documento se llevó a cabo un análisis comparativo de las poblaciones aspirantes y admitidas desde una perspectiva de género. A partir del examen de admisión que evaluó cinco componentes (matemáticas, ciencias naturales, ciencias sociales, análisis textual y análisis de la imagen) se supone garantizar un proceso de admisión equitativo y que sea para todos. 
Las informaciones regionales revelan que las mujeres optan preferentemente por las áreas correspondientes a Educación, Bellas artes y Ciencias sociales. Sin embargo, en los últimos años se han experimentado algunos cambios. Un caso excepcional es Cuba: en 1988 el 55,3 \% de los graduados en Ciencias exactas y tecnología eran mujeres (Bonder, 1994, p. 1).

Es común que los términos de equidad e igualdad de género sean confundidos. De ahí surge la necesidad de revisar su definición. En primera instancia, la Unesco (2014), como se citó en Unesco (2016), declaró que la igualdad de género es un principio relativo a los derechos humanos, un prerrequisito para un desarrollo sostenible centrado en las personas y un objetivo en sí mismo. Igualmente, el Fondo de Población de las Naciones Unidas (UNFPA) indicó que la igualdad de género parte de la idea de que todas y todos somos iguales en derechos y oportunidades. Así pues, la igualdad de género implica que féminas y varones tengan las mismas posibilidades $\mathrm{u}$ oportunidades en la vida en cuanto a acceder a recursos y bienes valiosos desde el punto de vista social y, asimismo, la oportunidad de controlarlos. En contraste, la equidad de género supone el trato imparcial entre féminas y varones, de acuerdo con sus necesidades respectivas. Ahora bien, con frecuencia, para asegurar la justicia, deben adoptarse estrategias y medidas para compensar las desventajas históricas y sociales, en el que la equidad es el camino hacia la igualdad (UNFPA, 2005).

No obstante, se puede observar en los hallazgos del estudio de Caro la forma paradójica en que las dificultades de admisión a la universidad son mucho mayores para ellas, a pesar de tener perfiles iguales a los de ellos. Con base en lo anterior, Quintero (2017) aseveró que "la mayoría de las mujeres tiene un puntaje menor al de los hombres en matemáticas y 
análisis de imagen, por lo que no alcanzan a ser admitidas en la Universidad". Además, determina habilidades bajo el sesgo de género a partir de un examen que evidentemente va en contravía de la apuesta "equidad para todos". Las dificultades y desigualdades en los contextos educativos tienen sus orígenes desde los primeros pasos por la academia tanto a nivel nacional como internacional.

Asimismo, lo demuestra otro estudio realizado por el estudio de Instituto Nacional de las Mujeres sobre féminas y educación en México (2015), el cual resalta que más de 2,5 millones de niñas no tienen instrucción escolar. Sin duda, este panorama da cuenta de una realidad de mayor vulnerabilidad en sectores donde las adultas mayores o indígenas son las más afectadas, ya que presentan una tasa de analfabetismo superior al 33 \%. En este mismo sentido, ocho de cada 100 mujeres mayores de 14 años no saben ni leer ni escribir mientras que más del $90 \%$ de madres adolescentes no asisten a la escuela.

En cuanto al contexto colombiano, desde los años treinta, la presencia de la mujer poco a poco se amplió en los escenarios educativos de la ES. No obstante, para esas pioneras, el escenario se revestía de diversas ambigüedades: reinaba el paternalismo y la galantería en las relaciones de género; la hostilidad de algunos profesores para quienes el saber era cosa de hombres; la ausencia de sanitarios para féminas; la expedición de los títulos en el universal masculino, por ejemplo, doctor en Medicina o en Derecho. Para la década de los años 6o, según lo declaró el Departamento Nacional de Estadística (DANE, 1970), tanto hombres como mujeres han tenido una participación similar: hombres el 68,6 \% y las mujeres $68,5 \%$, respectivamente (p. 3). Lo cual puede ser un indicador de un número similar en la población masculina y femenina entre los siete y los catorce años. 
Sin embargo, no se trata solo de acceder al sistema, sino de permanecer en él, sobre todo, incorporar las diferencias entre lo rural y urbano. Según Rama (1968), más del 14 \% de la población era urbana, mientras que el $41,3 \%$ era de origen rural. $\mathrm{Al}$ analizarse, estos datos evidencian una suerte de discriminación del sistema educativo colombiano, en el que sólo el 9,7\% de niños en las áreas rurales logra terminar el ciclo de básica primaria.

En cuanto a la educación media, el DANE en 1968 reportaba que la diferencia entre ellos y ellas que se involucraron en este marco fue tan solo de $1 \%$. Debido a que los matriculados fueron $49,5 \%$ mujeres frente a 50,5\% hombres. De este modo, puede decirse que la discriminación va más allá del género, debido a que las cuestiones de acceso respecto a quienes pueden matricularse o no y, a su vez, mantenerse en sus espacios de estudio, son también una forma de desigualdad. En cuanto a diferencias de rendimiento académico entre mujeres y hombres, no cabe duda de que es un tema que está ampliamente documentado. Se apunta a que, además del sesgo por habilidades en ciertas disciplinas, también se pueden observar factores socioculturales que influyen sobre estos grupos (Coronado et ál., 2012). En este sentido, la literatura permite comprender que esta problemática trasciende culturas.

Por ejemplo, las autoras López y Escalante (2016) detectaron la persistencia de violencia de género entre estudiantes universitarios españoles y mexicanos y el personal académico. Se concluyó que este último variaba el trato hacia sus estudiantes dependiendo del género. Entonces, al igual que Caro (2017), los principales aportes de estas investigaciones radican en la identificación de un problema de falta de equidad de género que se sostiene y reproduce en la educación de todos los niveles hasta llegar a la universidad y que, lastimosamente, prevalece en 
diferentes países. La desigualdad de género se convierte entonces en uno de los retos más importantes que enfrentan las IES. No obstante, no se puede ignorar que las luchas y exigencias del siglo XXI han traído una mejora para el ingreso a la ES de las féminas de forma progresiva (Alvarado, 2004). En la práctica, la inequidad de género persiste, no sólo en relación con el acceso, sino también tiene que ver con los nombramientos, las promociones o ascensos y evaluaciones propios del ámbito académico, que también se ven salpicadas por situaciones de violencia de género. Estas son visibilizadas en las diversas circunstancias de acoso y hostigamiento a la que se ven ellas permanentemente expuestas.

Si bien es cierto que las mujeres con estudios universitarios hoy en día incrementan su participación en diferentes contextos en todos los países del mundo, también es cierto que su ascenso ha sido complejo y, en muchos casos, frustrante (Eagly y Carli, 2012). En general, se tiende a suponer que el hostigamiento y el acoso son las únicas formas de violencia de género a las cuales se enfrentan las féminas en los escenarios de la ES. Sin embargo, estudios como el de Quintero y Caro (2017) dan cuenta de un tema "incómodo" que durante muchos años ha sido obviado y que, a su vez, señala una problemática que es una realidad no solo en Colombia o Latinoamérica, sino también en el mundo. Esta situación se ve atravesada por situaciones sociales, económicas, de clase, generación, raza y, particularmente, en función de género.

Por ejemplo, Caro (2017) exhibió en su tesis las primeras aproximaciones a la exclusión de las mujeres como un problema en el proceso de admisión de la Universidad Nacional de Colombia (UNAL), lo cual puede entenderse como un mecanismo de reproducción de los órdenes sociales y de género que podrían ser revisados también en las demás IES. Por lo tanto, los 
temas de discriminación, desigualdad y violencia de género en la ES se configuran como uno de los grandes desafíos a superar, puesto que los planteamientos en relación con la equidad de género pretenden ser principios fundamentales para el acceso a la educación.

Las féminas han sido abocadas a ejercer labores y profesiones del cuidado (enfermeras, manicuristas, amas de casa, niñeras, docentes). Esto lo demuestra el DANE (2020) cuando aseguró que en Colombia sobre el campo de la educación y la atención humana ocupan un 59 \% frente a los hombres, así como las mujeres realizan el 76,2 \% del trabajo de cuidados directos en el interior de los hogares sin remuneración económica; cifra que se resume en que el $91 \%$ de la población colombiana la mujer ejerce el rol de empleada doméstica. Por su parte, los hombres han acaparado las carreras STEM (Ciencias, Tecnología, Ingeniería y Matemáticas). En Colombia, para el mes de junio del presente año (DANE, 2020) respecto a actividades de comunicación e información, el hombre ocupa un $61 \%$ como un $91 \%$ en el campo del transporte. En estas carreras se asignan y exigen características como la integralidad (inteligente, atractivo, y caballero) independencia, fuerza, racionalidad, orientación a la consecución de metas y la competencia como forma de supervivencia. Aunque muchas universidades han empezado a establecer protocolos, campañas políticas, públicas y programas para alcanzar la equidad. Asimismo, han orientado sus esfuerzos en superar la discriminación, el acoso y otras formas de violencia de género, pero estas siguen reproduciéndose en el mundo contemporáneo. De este modo, podría decirse que un diagnóstico como el que presentó el Observatorio de Asuntos de Género de la Universidad Nacional de Colombia a partir de la tesis de Caro, es un llamado a incorporar la perspectiva de género en los procesos de admisión de las IES. En ese sentido, los diagnósticos que 
puedan generarse sobre la condición de igualdad entre hombres y mujeres de cada institución, podrían ser analizados para homogeneizar criterios que permitan generar políticas institucionales que en el mediano y largo plazo puedan garantizar la participación con equidad de género en los distintos escenarios de ES. Así las cosas, no solo se garantizaría la paridad de género en las convocatorias y admisiones, sino que también impulsaría el acceso al trabajo, el desarrollo profesional y el fomento de la investigación para ellas.

En este sentido, la constante evolución del mundo contemporáneo y la incorporación de las TIC en la educación exigen, especialmente en contextos de ES, promover espacios de equidad, igualdad e inclusión para todos. Sin embargo, como mencionó, la distribución desigual de la ES sigue siendo una constante. Es así como en términos de acceso, permanencia y movilidad, la exclusión es el común denominador, en particular para las mujeres y, aún más, si ellas quieren vincularse en disciplinas relacionadas con el mundo de la ciencia y la tecnología. No se puede ignorar que las brechas digitales de género son ahora una apremiante realidad que se anexa a las otras desigualdades ya mencionadas y, por consiguiente, van más allá del género. Esta idea está relacionada con la ausencia de competencias digitales, formación en servicios TIC tanto para la formación como para la investigación. Esto brindaría también oportunidades de liderazgo y emprendimiento en ofertas de trabajo en escenarios del sector digital. Todo lo anterior requiere de participaciones femeninas en STEM que ayuden a derrotar los sesgos de género acerca de las mujeres en los retos de la sociedad del mundo de hoy que tiene todo que ver con la articulación de la relación de género y TIC. 
En síntesis, reflexionar en la manera de diagnosticar y sobre qué tan equitativo es el acceso a las IE puede ser un primer gran paso. Por ejemplo, las coordenadas de género del mundo contemporáneo están definidas por fenómenos tan diversos como la masculinización de la cultura laboral en corporaciones transnacionales y multinacionales (Connell, 2014). Por lo tanto, la organización social del mundo contemporáneo, las categorías femenino-masculino, la incorporación y el uso de los artefactos tecnológicos en la educación y la propia humanidad engendran a diario desigualdades que podrían ser un objeto de observación para una futura investigación. En esta podrían articularse las políticas de igualdad de oportunidades para la mujer en los ámbitos de la educación. Claro está, se debe pensar en tres momentos desde una postura feminista en el que ni las situaciones de tensión, ni las implicaciones epistemológicas y políticas que esto genera puedan ser obviadas.

\section{Desafíos TIC y equidad de género}

La tecnología no es neutral, está dentro de lo que hacemos, y está dentro de nosotros.

Vivimos en un mundo de conexiones e importa cuales se hacen y deshacen.

Donna Haraway

Es importante aclarar que la comprensión del sesgo de género para las mujeres en la tecnología es también tratar de descubrir que, desde que se menciona la desigualdad de género en diferentes escenarios, estos siempre terminan siendo un conductor de tensiones, discusiones, intolerancias, tiranías y debates. La sexualidad y el género desde la interseccionalidad 
han puesto en evidencia el modo en que las formas de las relaciones de poder y la dominación social están profundamente vinculadas. Aunque desde hace algunos años la interseccionalidad se ha convertido en la expresión utilizada para designar la perspectiva teórica y metodológica que busca dar cuenta de la percepción cruzada o imbricada de las relaciones de poder, el modelo predominante de dominación masculina sigue siendo la característica por resaltar de los últimos siglos.

Desde hace más de quince años el mundo ha estado inmerso en una suerte de "boom" en torno al uso de las TIC. De acuerdo con Castells, como se citó en Silvela (2004), se determina una nueva era acompañada por varios fenómenos de trascendencia histórica denominada "La era de la Información”. En esta se modifican las bases materiales de la sociedad, el modo en el que los hombres se relacionan entre sí y con su entorno, así como sus modos de producción y de consumo. Estas transformaciones sociales cotidianas no se han logrado de igual forma entre grupos socioeconómicos o a nivel de género. Así pues, quienes tienen mayor poder adquisitivo poseen además un mayor acceso a las TIC (Cabero y RuizPalmero, 2018), en el que se origina la brecha digital.

La discriminación de género ha sido una característica permanente a lo largo de los acontecimientos de la historia. La era de la revolución digital también la ha heredado como producto de sociedades patriarcales y desiguales que aún están en busca de la igualdad de género. Dentro de este marco se encuentra la "brecha digital de género" caracterizada por: "patrones de segregación sexual que se reproducen en la economía de la información, nuevas desigualdades entre las mujeres en empleos relacionados con las TIC y participación de mujeres en el teletrabajo muestran las nuevas facetas de sobre explotación” (Bonder, 2006, p. 8). Por lo tanto, un reto del mundo de hoy consiste en desarrollar un modelo 
social que garantice el acceso y uso de las TIC a las féminas, una apuesta de democratización de la educación y de la promoción de la participación de las mujeres en la producción de conocimiento. La inclusión de ellas en las TIC podría hacer una gran diferencia en la disminución de la brecha digital de cara al futuro.

En cuanto a la brecha digital, para Compaine (2001), autor del libro The Digital Divide, este concepto puede entenderse como la división percibida "entre aquellos que tienen acceso a las tecnologías de la información y la comunicación contemporáneas y aquellos que no, donde quienes no tienen acceso, terminan quedándose en una evidente desventaja económica y social respecto de los demás" (p. 32). De manera general, el factor determinante para medir la brecha digital se fundamenta en la falta de acceso a internet, ya sea por la ausencia de conectividad, la mala calidad de esta o por el desconocimiento en su uso. Estas características son propias de la región por su ubicación geográfica y por razones de desarrollo político-social, en los que se percibe que la conectividad sigue siendo uno de los retos más grandes.

A lo anterior, se suma lo que dijo ONU (2020), a partir de la crisis presentada por la pandemia mundial: el cierre de escuelas en trece países para contener la propagación de COVID-19 ha precipitado un cambio radical y tecnológico en toda la dinámica mundial. No se puede ignorar que la ES fue uno de los sectores que ha experimentado más impacto y transformación digital. La educación está convocada a la utilización de las TIC y, en esta misma vía, los docentes y estudiantes deben afrontar nuevos retos profesionales en constante cambio, donde "la adaptación a las circunstancias obligará a tener una mente abierta y la información circulará por doquier" (González, 2016, p. 26). En definitiva, esta es una de las consecuencias innatas de la evolución de las TIC y los efectos negativos 
del COVID-19, lo que obvia las experiencias, trayectorias y realidades de quienes asisten a la academia y, aún más, de las mujeres que aprenden o enseñan de cara a estos asuntos.

En este sentido, la incorporación, uso y acceso a este tipo de tecnología no siempre está al alcance de quienes enseñan (por las distancias generacionales), quienes se forman (por cuestiones de enfoque), las IES que forman (oficiales-privadas) y las brechas digitales que se visibilizan (por razones de género, edad, raza, clase, entre otros factores). Estas desigualdades pueden ser detonantes de factores de exclusión y que llevan detrás una construcción histórica de roles para los géneros que no están relacionadas con las capacidades individuales de las mujeres (Romero, 2018). El acceso de ellas al conocimiento y a las TIC son esfuerzos insustituibles para quienes aspiran a contribuir a superar los problemas causados por la desigualdad de género. Esto ha sido ratificado en las Metas 2030 para América Latina (CEPAL, 2018), en el que se estableció el presente fin: "lograr la igualdad de género y empoderar a todas las mujeres y las niñas, mejorando el uso de la tecnología, en particular la tecnología de la información y las comunicaciones, para promover el empoderamiento femenino".

Así, de manera muy sutil, la feminización de los estilos de liderazgo, la redefinición de las jerarquías y el poder adquisitivo de las féminas ha desplazado milimétricamente del centro de poder los roles y actitudes (sobre todo masculinas) frente a lo que se identifica hoy como trabajo y familia. Estos dos últimos se han concebido históricamente como conceptos separados, en el que el hombre se ocupa de lo primero y la mujer de lo segundo, como si las implicaciones epistemológicas y sociales les impidieran una sana convivencia. Si uno de los objetivos de la educación es formar a los estudiantes para enfrentarse a la complejidad de 
problemas propios del mundo y de la sociedad en general, entonces es prioritario promover entre los estudiantes la capacidad para pensar de manera lógica y crítica. Así pues, buscar alternativas para adoptar diferentes enfoques sistémicos en cuanto a la toma de decisiones que reflejen la realidad fuera del aula (Andenoro et ál., 2017; Haber-Curran, Paige y Tillapaugh, 2013, citados por Díaz, 2020). En suma, una realidad atravesada por temas de equidad de género.

No obstante, según Mancilla et ál. (2019), son varias las investigadoras que se han referido a las TIC como "un nuevo club de hombres". En otros términos, un mundo dominado por parámetros masculinos que pretende que las mujeres se adapten a las tecnologías tal cual están planteadas. Lo anterior sucede sin que se consideren, en muchos casos, puesto que su configuración responde netamente al mundo simbólico masculino; situación que puede seguir reproduciendo desigualdades. Aunque la igualdad de oportunidades entre mujeres y hombres ha sido una prioridad para todos los aspectos de desarrollo del país, existe una baja representación de la mujer en todos los sectores que tienen que ver con el uso de tecnología desde la educación hasta el trabajo. La Comisión Interamericana de Derechos Humanos de la Organización de los Estados Americanos (CIDH/OEA) destacó que "entre las barreras persistentes en Latinoamérica, el propio sistema educativo puede constituir el principal obstáculo para lograr una educación en condiciones de igualdad”.

Por otro lado, las formas de organización del mundo como se conoce hoy se han visto permanentemente abocadas a extremos y vínculos entre lo femenino y lo masculino. De ahí que se perciban frases como "los hombres no lloran" y las mujeres "dicen que sí cuando quieren decir no". Sin embargo, la diversidad sexual y de género del escenario contemporáneo redefine la identidad de género con una perspectiva interseccional de 
liderazgo, que sobrepasa los condicionamientos de los roles o el aspecto físico, al sentir individual. Los líderes inclusivos, de acuerdo con los planteamientos de Fernández y Hernández (2013), son individuos que promueven la diversidad, equidad, la mejora continua, la confianza, el desarrollo profesional y el trabajo en equipo.

El conocimiento epistemológico ha de verse transformado en una realidad en el que la ciencia y la tecnología van cada vez más rápidos. Se abren espacios para la división digital (nuevas formas de nombrar la brecha digital), en el que los seres humanos más allá de su raza, género, condición y clase, deben diariamente enfrentar y buscar cómo superarla. La división digital es definida por Rogers (2001) como se citó en Castaño (2008):

La brecha que existe entre individuos que sacan provecho de Internet y aquellos otros que están en desventaja relativa respecto a Internet” y lo relaciona con la hipótesis de la brecha del conocimiento (knowledge divide), es decir: A medida que aumenta la difusión de los medios de comunicación de masas en el sistema social, ciertos segmentos de la población, con un nivel socioeconómico más elevado tienden a apropiarse de la información a una velocidad más rápida que los del nivel más bajo, y de esta manera la brecha entre estos segmentos tiende a aumentar en lugar de a reducirse. (párr. 14)

Aquí las cuestiones de interseccionalidad deben conducir a la construcción de propuestas situadas empíricamente en una situación problémica que refleje el porqué de los diferentes cruces de desigualdades, e inclusive de la brecha digital de género. Es fundamental precisar que se redefine si existe por razones de habilidades, de raza, de edad o clase, entre otras categorías, incluida la ubicación geográfica. En Colombia, a pesar del reconocimiento de la ciudadanía a partir de la diferencia cultural y de los derechos sociales, económicos y políticos, las desigualdades sociales son cada vez más 
profundas en el conjunto de la sociedad (Quintero, 2017). Al reflexionar sobre la clave interseccional, se debe permitir la aproximación a otra forma de identidad, igualdad y poder, y, de esta forma, sobrepasar el género o las categorías establecidas socialmente y que, en últimas, terminan siendo las que definen el acceso o no a diferentes derechos y oportunidades. De este modo, enaltecer y abrazar la diversidad para posibilitar el acceso a los procesos educativos en todas las instituciones. A pesar de estos datos, las mujeres siguen ocupando menos puestos de poder e incorporación en los ámbitos de la economía, la ciencia, la tecnología y, en general, de la educación. Por ende, la incorporación y uso de las TIC se convierte tanto en un reto como una oportunidad para ellas, puesto que, sin duda, ayudará a reducir la desigualdad de género y a reducir las distintas brechas digitales de género. Asimismo, Unesco (2019) declaró ocho perspectivas en las que es indispensable que las mujeres cuenten con competencias digitales:

1. Facilitan la toma de decisiones y se favorece la incorporación de las féminas a la actividad económica y el empleo.

2. Son esenciales para garantizar la seguridad de las mujeres tanto online como offline.

3. Favorecen la participación de ellas en la vida política y la comunidad.

4. Aportan beneficios económicos a las mujeres y a la sociedad.

5. Facilitan la incorporación de ellas en la educación online, a las posibilidades de emprender un negocio propio o incorporarse al mercado laboral.

6. Para que participen en el diseño de las tecnologías de manera que contribuyan al planteamiento de la OCDE (2018). Con ello pueden contribuir a cerrar la brecha salarial, debido a que las compensaciones 
para las mujeres con competencias digitales son mayores que las que se pueden obtener gracias a otras competencias.

7. Empoderan para la igualdad de género.

8. Aceleran el progreso de las mujeres hacia la consecución de los objetivos de desarrollo sostenible. Lo cual se encuentra contemplado en los Objetivos de Desarrollo Sostenible (ODS) específicamente en los Objetivos de Educación de Calidad (ODS 4) y de igualdad de género (ODS 5).

Las IES deben entonces promover la diversidad y la inclusión de manera permanente. Esto implica contar con equipos de trabajo compuestos por individuos con características y experiencias diferentes. Lo anterior promueve la diversidad de género, edad, lugar de origen, ideología y, a su vez, el fomento de prácticas directivas inclusivas que lleven a la participación de todos para potencializar sus diferencias (Sherbin y Rashid, 2017). De este modo, la experiencia educativa consolida las relaciones de equidad y solidaridad, en el que las mujeres puedan alcanzar la igualdad de género y contribuyen al desarrollo económico del país como agentes de cambio.

Para terminar este apartado se concluye lo siguiente: la digitalización y la imparable difusión de la automatización y la inteligencia artificial constituyen el gran reto del empleo presente y futuro, tanto en términos cuantitativos (cuántos empleos se crean y destruyen) como cualitativos (cuáles serán los nuevos empleos creados; cómo se transformarán los empleos que permanezcan). (Sáinz et ál., 2020, p. 15)

Entender la situación de las féminas hoy en día y de su constante lucha por la equidad de género, en escenarios donde las tecnologías de la información y de la comunicación se encuentran altamente relacionadas, se convierte en un aporte que, desde esta perspectiva, puede ser abordada 
en futuras investigaciones. Las cifras demuestran que más del 50 \% de la población mundial son mujeres y más de la mitad de las titulaciones universitarias son para ellas. Sin embargo, en la ES, la influencia de lo masculino se encuentra en las metodologías, los procedimientos, los temas seleccionados y el lenguaje utilizado (Gore, 1996).

\section{Pensando en clave interseccional}

No se nace mujer, sino que se llega

a serlo.

Simone de Beauvoir

En primera instancia, es vital citar el discurso pronunciado por Soujourner: “Ain't I a woman?”3 (¿acaso no soy una mujer?) en la Convención de Mujeres de Akron-Ohio en diciembre de 1851. Esta experta nació en 1797 bajo la esclavitud y dedicó parte de su vida a ser una abolicionista y activista por los derechos de la mujer. Ella acudió a los tribunales para recuperar a sus hijos y se convirtió en la primera fémina negra en ganar un juicio en contra de un hombre blanco. El nombre de nacimiento de Sojourner era Isabella (Bell) Baumfree, pero cambió su nombre a Sojourner Truth en 1843 que significa "residente temporal de la verdad”. Su discurso es uno de los primeros discursos públicos transmitidos oralmente, en el que se empiezan a plantear las primeras ideas sobre lo que posteriormente será la teoría de la interseccionalidad. En él realizó una crítica doble: al feminismo blanco, debido a que las mujeres negras esclavas no se sentían recogidas en él, y al movimiento abolicionista por mantener la diferencia entre varones negros y féminas negras.

${ }^{3}$ Interpretación del discurso: https://www.youtube.com/watch?v=eUdxsQ0Qsrc 
Posteriormente, en los años setenta surgió la organización feminista negra Colectiva del río Combahee, liderada por la primera guerrillera americana Harriet Tubman, que dirigió una acción de liberación de más de 750 esclavos en Carolina del Sur. La Declaración de la Colectiva del río Combahee (Combahee River Collective Statement) empieza a reivindicar el pensamiento negro feminista con la recuperación de discursos e historias como el de Sojourner. Esta declaratoria permite ubicar una primera formulación de la teoría de la interseccionalidad y es considerada como un manifiesto político desde el pensamiento negro feminista, el cual trascendió lo político hacia la academia.

El documento se encuentra dividido en cuatro partes: 1) génesis del feminismo negro contemporáneo en el que se reconoce el legado de mujeres que no son conocidas, pero que reconocen la identidad sexual e identidad racial como situaciones vitales que sirven para enfocar batallas políticas. 2) Postura política y de creencias del colectivo, en la que se declara su valor propio e independiente como reclamo de devolución de su humanidad, igualdad y libertad como mujeres negras desde su propia política de identidad propia. 3) Problemas históricos en la organización de las feministas negras, en dicho apartado se propone la no segregación en la organización política y, en consecuencia, la ampliación del feminismo negro a otros feminismos "del sur". 4) Propuesta de temas y práctica feminista en el que se incluyen preocupaciones por los derechos sexuales y reproductivos, así como el acceso a servicios de salud. Además, políticas concretas para la lucha en contra de desigualdades generadas a través del racismo, clasismo y sexismo como los grandes sistemas de opresión. Claramente, las subjetividades en relación con el tema y que configuran la actualidad son construidas en forma macrosocial, mientras se observa el desarrollo de habilidades que permiten ver o no algunas cosas. Esas 
“cosas" prevalecen en el marco de dos tensiones configuradas por la sociedad, en términos de lo que es y hace hombre y lo que es y hace una mujer. Y es que lo que no se puede ver, eso que no se puede describir en términos de igualdad de género persiste en el "estar ahí", en el que quiera que se piense ese "ahí".

Por lo tanto, la perspectiva interseccional surge como tejido social que, a su vez, es un tejido construido de forma interseccional. En este se subraya el carácter diferenciador de lo personal, de lo social, la centralidad de la relación interespecies como una perspectiva epistemológica relacional que invita a salir del individualismo metodológico. Así pues, se resalta la importancia y pertinencia de la relación, los vínculos e inclusive de la interdependencia. En este sentido, la teoría propone que se debe pensar en cada elemento o rasgo de una persona como unido de manera inextricable con todos los demás elementos, con el fin de comprender de forma completa la propia identidad.

Una primera noción de interseccionalidad está elaborada a partir del estudio de "la gente oprimida", es decir, de aquellas oprimidas por el género, por la raza y por la clase $\mathrm{y}$, asimismo, las intersecciones que se dan entre estas. Otra noción se aleja del estudio de los grupos sociales para pasar al de las relaciones de dominación y, de este modo, reflexionar la interseccionalidad desde una perspectiva de la experiencia. Esta última tiene en cuenta que todas las personas se encuentran atravesadas por el género, la raza y la clase, es decir, todas están en la intersección de esos tres sistemas, inclusive aquellos que son dominantes o que ocupan un lugar de privilegio. De esta forma, la interseccionalidad se puede entender como esa imbricación de sistemas de dominación y de opresión, en el que todas las personas viven de manera diferencial esos mismos sistemas de dominación. 
Esa segunda noción de interseccionalidad está implementada por los estudios críticos que se preguntan por la construcción social ideológica e histórica de la raza, a partir de la cual se construyen categorías de dominación como "indio" o "negro". Asimismo, el análisis relacional de la dominación implica contemplar categorías dominantes frente a las de dominación, por ejemplo, la categoría dominante en raza sería "lo blanco". Pensada así la interseccionalidad, se entiende que las categorías tienen un carácter político y que, por lo tanto, se convierten en campos sociales en disputa en sí mismas.

Ahora bien, el racismo cotidiano en universidades puede ser entendido entonces como un proceso social. Incluso, es preciso considerar la actual situación de pandemia por el COVID-19, la cual ha renovado en grandes proporciones el racismo en el país. No es un secreto afirmar que la revolución digital transforma el mundo como se conocía, lo que ha acelerado la competitividad y producción de conocimiento. En este sentido, sin una alfabetización digital suficiente y pertinente para las necesidades de ese mundo, otras desigualdades se visibilizan. Esto hace que de forma simultánea, se incluyan y excluyan a los miembros de la sociedad a partir de la brecha digital. Las desigualdades que genera el avance significativo de la tecnología, igual que cualquier otro avance en la historia, dejan en riesgo a muchas personas, por razones de estatus, género.

Mirándolo así, excluye a la población, limita el acceso, la incorporación y uso de las TIC. Lo que, por obvias razones, empieza a dejarles fuera de oportunidades para el futuro. Por ende, quienes tengan acceso o no y aquellos que usen la tecnología o no, quedan rezagados en una sociedad altamente tecnificada. Esto resulta interesante desde la teoría de la interseccionalidad para incluir una perspectiva situacional, la cual debe 
combinar lo estructural y lo situacional como elementos para comprender las desigualdades sociales y así poder también abordar la brecha digital de género.

Hay entonces un gran reto, puesto que la trilogía (clase, género, raza) quizás no sea la verdad de oro para abordar. Connell (1995) señaló que el género, como estructura que ordena la práctica social, interactúa con otras estructuras sociales, por ejemplo: raza, clase, nacionalidad o posición en el orden mundial. "Para entender el género, entonces, debemos ir constantemente más allá del género", ya que "las relaciones de género son un componente importante de la estructura social como un todo" (Connell, 1995, p. 76). Aquí, las relaciones de poder y el capitalismo, generalmente patriarcal y racista, serían entonces conceptos imbricados que deben incomodar y llevar a posturas feministas. Es vital que interpelen los conocimientos de todas las disciplinas para, desde ahí, tratar de comprender la participación de las mujeres en el uso de las TIC en la ES. Las desigualdades que se (re)producen en el mundo contemporáneo a partir de la incorporación, uso y acceso a las TIC, dejan entrever que la utilización de internet por parte de los ciudadanos es desigual (Norris, 2001; Van Deursen y Van Dijk, 2014 citados en Herranz et ál., 2017). Sobre todo, en estos tiempos donde la crisis por la pandemia, esto ha descarnado una realidad en torno a brechas de género y digitales de género que conllevan grandes desafíos para las IES, que se evidencia en la manera de garantizar procesos de admisión y permanencia en términos de equidad e igualdad de género. Según el informe de Género y TIC de Gurumurthy (2004), existe un cambio en el curso de la sociedad a partir de las innovaciones tecnológicas y en relación con el debate sobre la igualdad y la justicia en el ámbito del derecho de las féminas al acceso igualitario. 
La utilización y la administración de los espacios digitales hace hincapié respecto a dos momentos de la historia entre pre- y posinternet, en el que se formula una propuesta teórica sobre las relaciones que ellas enfrentarán en un mundo laboral altamente tecnificado. Esto está sucediendo en este preciso momento, de este modo que es obligatorio contar con nuevos enfoques y metodologías que empezaran a dar cuenta de una sociedad de la información incluyente, democrática y con justicia de género. Sin embargo, en este marco sólo sería posible construir desde la sociedad civil y la academia.

La literatura revisada hasta el momento da cuenta de lo que es la realidad, en cuanto a las tensiones, retos y oportunidades que enfrentan todos los colectivos, asociaciones comunitarias e individuos que pugnan por un uso de las TIC y de internet libre, neutral y accesible. El ideal se fundamenta en derribar estereotipos y redefinir las relaciones de poder; se democratice la exposición de la diversidad cultural y se abran espacios equitativos para la reflexión sobre múltiples temas relacionados con las dimensiones de igualdad, género y diversidad social y cultural. Estos elementos pueden ser considerados desde la interseccionalidad, la cual puede ayudar a dar cuenta de la no homogeneidad de las mujeres en la ES, puesto que requiere aprehender las relaciones sociales como construcciones simultáneas en distintos órdenes en el que coexisten múltiples dimensiones de la opresión, exclusión y la marginación (Viveros, 2016). Queda claro que durante los últimos años se ha construido un destino social. Pero también es evidente que, para el mundo globalizado actual, hay que ir más allá de las razas y las cuestiones de género, en el que se conceptualice la diferencia de género como un instrumento y artefacto del dominio masculino. Las diferencias son naturalizadas para reproducir las desigualdades de clase y esa naturalización es un producto ideológico. Así, 
hay unos grupos que son más iguales que otros, e incluso hay vidas que importan más que otras. La realidad que se enfrenta hoy en día frente a la pandemia ha puesto en evidencia lo que ya existía: una democracia que celebra igualdad de oportunidades, pero que aún excluye. ¿Cómo es posible que, al ser todos humanos, se mantengan estas agencias frente a los iguales?

Al ser Colombia un país multicultural, la interseccionalidad aparece entonces como herramienta en el que hablar de mujer y tecnología puede propiciar un entorno que esté centrado en las personas, la comunicación y la cultura para que las féminas prosperen. Esta sería la implicación epistemológica y política que, además, no se limita a los campos feministas, sino que interpela de formas particulares la situación de la academia latinoamericana y de otras regiones no centrales a la producción del conocimiento. A diferencia de hace un tiempo atrás, en el presente se puede ser globales en escenarios análogos y digitales, en el que la competitividad ya no es de permisión exclusiva de entornos grandes, blancos, masculinos y bastante homogéneos del pasado, exclusivos de personas procedentes de un contexto económico, educativo y familiar específico.

Aunque la brecha digital de género sea una constante en el tiempo, las mujeres también están decididas a adaptarse y no morir en este intento. "A veces para resistir, uno tiene que irse, otras, uno tiene que quedarse; jugar el juego. No para obtener el reconocimiento en las lógicas neoliberales del sistema, sino porque se desea tener una conversación horizontal" (Viveros, 2016, p. 1).

Aunque el concepto de la interseccionalidad se ha posicionado como un referente teórico contemporáneo en los asuntos de género, el asunto que refiere a cómo "estudiar a las mujeres". Esto es aún algo nuevo y en 
construcción, puesto que no existe un consenso respecto de la manera de abordarla, dado su carácter interdisciplinario y su aplicabilidad a varios propósitos. (Brah, 2013, p. 18)

$\mathrm{Al}$ respecto, McCall (2005) planteó la interseccionalidad como un reto metodológico en el que el sujeto de análisis puede incluir varias dimensiones y entonces no se puede abordar desde una sola mirada, puesto que se podría caer en el reduccionismo o la simplificación.

Como cualquier herramienta analítica, esta se ve condicionada al uso que pretenda dársele y, de este modo, así evitar en su aplicación como una suerte de justicia social, no caer en nuevas injusticias. Tal como lo advirtió Lugones (2005): la interseccionalidad no resuelve el problema, solo lo muestra. Por lo tanto, no se trata solamente de utilizar el término "interseccional", sino de situarse en una genealogía determinada que abarque los problemas de igualdad y las diferencias con sus relaciones con el poder, en el que se puedan observar los riesgos, impactos, experiencias y necesidades diferenciadas de todas las personas.

En este sentido, los indicadores de inclusión social y equidad deberían permitir, en términos generales, una aproximación a un aspecto de la realidad social. Claro está, se debe entender sus diversas tipologías de clasificación entre lo cualitativo y lo cuantitativo, lo absoluto y lo relativo, lo simple y lo compuesto. "Gracias a la información que estos indicadores proporcionan, se pueden hallar respuestas a problemas sociales concretos y a tomar decisiones de políticas públicas sustentadas en la evidencia empírica" (Cecchini, 2005, p. 11). En este sentido, las iniciativas de medición de equidad de género a nivel internacional son variadas y muchas.

Según se mencionó en la introducción, desde Cooper (2015), Collins y Bilge (2016) se reconocen seis principios sobre la interseccionalidad como 
herramienta analítica: 1) la desigualdad social para la cual el cruce, clase social, género y raza son generadores predominantes de dominación4; 2) la realidad social debe ser entendida desde las relaciones de poder que la sostienen; 3) se debe evitar la carnalización de situaciones binomiales a partir del pensamiento relacional; 4) es indispensable proporcionar una mirada interseccional como una suerte de contextualización; 5) se deben comprender los fenómenos sociales desde cómo se da la intersección (clase, raza, género, etc.) más allá de entenderla solamente como manifiesto de opresión; 6) y asociar la interseccionalidad como un proyecto orientado a la justicia social. De este modo, es posible reconocer que tal vez muchas investigaciones se han abordado desde este concepto sin haberlo definido como tal, lo cual puede favorecer el diálogo entre diferentes problemáticas 5 inter, trans y multidisciplinares.

Un caso ilustrativo es la propuesta de Social Watch (2009) sobre el Índice de Equidad de Género (IEG) para medir las dimensiones sociales, económicas y políticas de género. También, la propuesta del Foro Económico Mundial (2006) con el índice Global de la Brecha de Género, el cual aborda la distribución de los recursos económicos, nivel educativo alcanzado, la tasa de supervivencia, la salud y la participación política. Seguido de esto, encuentro el índice de Instituciones Sociales y de Género (SIGI), conformado por la Organización para el Desarrollo Económico (OCDE), que incluye aspectos cualitativos, por ejemplo: relaciones patriarcales, composición familiar, entre otras. Igualmente, el Programa

\footnotetext{
4 "Hablar de matriz de dominación remite al trabajo de la socióloga afroamericana Patricia Hill Collins (1990) que, con esa metáfora de matriz, quiere que se comprenda que la dominación y la resistencia se construyen y moldean en un cruce" (Martínez, 2020, p. 285).

${ }^{5}$ En el sentido amplio del término, como casos, problemas, realidades sociales, malestares, aquellos que aquejan a largos sectores de la población y tienen que ver con las condiciones objetivas y subjetivas de vida en sociedad. Sus causas pueden hallarse en aspectos económicos, políticos, entre otros.
} 
de las Naciones Unidas del 2010 para el Desarrollo (PNUD) con el índice de Desigualdad de Género que combina medidas de bienestar y potenciación de género desde tres perspectivas: salud, empoderamiento y participación en el mercado laboral.

En cuanto al contexto latinoamericano, la Comisión Económica para América Latina y el Caribe (Cepal) (2007) creó el Observatorio de Igualdad de Género de América Latina y el Caribe. En este buscó proporcionar a los países miembros indicadores estratégicos de género y variadas herramientas analíticas para la formulación de políticas públicas. Como se puede observar, son muchas las organizaciones que han emprendido una campaña para diagnosticar, analizar y revisar a profundidad las cuestiones de género en el mundo. A pesar de eso, la ausencia de estadísticas especializadas en torno a estos asuntos es una realidad. Por lo tanto, abordar de manera completa la complejidad de las desigualdades de género es un deber ser en los procesos investigativos a los que clama el mundo de hoy. Sumado a esto, los desafíos a los que se enfrentan las Instituciones de educación de Colombia sobrepasan los indicadores de género. En un principio se empieza por la necesidad de definir con claridad a que se refieren las instituciones en términos de equidad, igualdad e inclusión.

De este modo, la historia de la mujer en la educación colombiana es uno de los logros más importantes de la Colombia contemporánea. Han pasado más de 83 años desde que la primera colombiana entró a la universidad. La presencia de la mujer en estos escenarios permite visibilizar diferentes tipos de violencia y desigualdad que pueden ser interpretados desde diferentes perspectivas científicas, mientras abren camino para futuras generaciones y abordajes de estudio que trascienden de lo académico a las leyes $\mathrm{y}$, por ende, a la vida. 
Recordar las múltiples experiencias actuales que permiten a todas las mujeres un acceso a la ES menos masculinizado, es un llamado a tener siempre presente y, asimismo, identificarlas como personas situadas desde unas experiencias concretas. Al realizar dicha estrategia de forma explícita en las diversas indagaciones e investigaciones, sin duda, es una muestra de honestidad y objetividad. Desde la pedagogía feminista, en diálogo con las pedagogías críticas, se abre el espacio para fomentar escenarios de libertad en el que se abre el espacio para otras pedagogías que rompan las barreras invisibles propias de la historia.

De modo que, si se aprecia y reconoce la diversidad femenina, inter, multi y pluridisciplinar, inquieta, como cúmulo de interseccionalidades, incluso más allá del género, se generan conexiones entre disciplinas y variedad de temas. Desde esta perspectiva, es un deber el desnaturalizar la raza, la clase y el género y, asimismo, seguir resistiendo a las colonialidades como ejercicios epistemológicos, metodológicos y empíricos, sobre todos a estos sistemas de dominación que atraviesan y heredan permanentemente la historia y que terminan siendo incorporados en los semilleros, proyectos y procesos de investigación.

Finalmente, diagnosticar es apenas el principio de un universo que debe ser analizado, estudiado y comparado entre países de América Latina. Así pues, la idea consiste en trascender la estadísticas y datos que se centran en procesos de matrícula escolar a permanencia y trayectoria en los estudios. Incluso, en estos tiempos de crisis, la brecha de género y la brecha digital están aún más visibilizadas, no solo con respecto a los aspirantes, admitidos en procesos de ES, sino también en la comunidad académica en general con una mirada especial a los docentes. Son las comunidades de aprendizaje colectivo y las alianzas de cooperación mediadas por la tecnología, una gran oportunidad de reconocimiento a la 
interdependencia que puedan ayudar de manera sostenible a la aseguranza de los procesos de educación para todos.

La relevancia y pertinencia de una apuesta investigativa con una perspectiva interseccional podrían concebirse como un espacio de información y comunicación de experiencias y debates. En estos últimos se abordan temáticas de inclusión social y equidad en la ES; asimismo, se evalúan opciones de oportunidad para superar desigualdades sociales que sistemáticamente se relacionan con la brecha de género y por la presencia permanente de la mediación tecnológica en la educación que ahora también abre la puerta a la brecha digital de género.

En definitiva, la educación debe ser sinónimo de desarrollo productivo con equidad para las mujeres en el mundo contemporáneo. Debe asegurar el acceso, la calidad y la inclusión como derroteros de la desigualdad. En este sentido, teorías como la de la interseccionalidad permiten construir la realidad a partir del cuestionamiento de los procesos sociales y culturales $\mathrm{y}$, asimismo, no solo naturalizarlos. Al categorizar la cotidianidad y sus significados, se debe profundizar en sus significados. Con esto, se podría desmontar y actualizar los "falsos consensos" de que las categorías son monolíticas y universales. Entonces, las identidades no son tan estables y fijas como se piensa y, por ende, han de entenderse en relación con otras formas estructurales de desigualdad, en el que la clase social, la sexualidad, el acceso a la educación, entre otras, están directamente aludidas e imbricadas en las posibilidades de mejora de calidad de vida de las féminas.

De ahí la importancia de pensar en clave interseccional, para no generar una "única" interpretación desde la posición situada de quienes interrogan la realidad desde diferentes frentes, puesto que se incluyen los propios sesgos e intereses. Finalmente, para superar las desigualdades más allá del 
género, se debería hacer énfasis en opciones educativas y ocupaciones en todos los momentos educativos: generar oportunidades más allá del género. Esta idea implica reevaluar las áreas que se han considerado tradicionalmente femeninas, lo que hace visibles los interés y perspectivas de las mujeres desde la educación preescolar hasta la ES en el que se promueva la igualdad de oportunidades en todas las disciplinas. Asimismo, es importante articular cambio de actitudes y cambio en las estructuras de poder de la mano de conocimientos en competencias digitales para optimizar el uso, apropiación e incorporación de las TIC, con el fin de superar los sesgos, la discriminación y las brechas de género.

\section{Referencias}

Alvarado, M. (2004). La educación "superior" femenina en el México del siglo XIX. Demanda social y reto gubernamental. CESU/Plaza y Valdés.

Arango, M. y Corona, E. (2016). Guía para la igualdad de género en las políticas y prácticas de la formación docente. Unesco.

Bonder, G. (1994). Mujer y educación en América Latina: hacia la igualdad de oportunidades. Revista Iberoamericana de Educación, 6, 1.

Bonder, G. (2006). Equidad de género y cultura de la innovación colaborativa: nutrientes para fortalecer la sociedad del conocimiento. Conferencia Internacional Know How.

Bourdieu, P. (2002). Interventions Politiques. Marsella.

Bourdieu, P. (2014). La distinción. Taurus.

Brah, A. (2013). Pensando en y a través de la interseccionalidad. En M. Zapata, S. García y J. Chan, La interseccionalidad en debate. Actas del Congreso Internacional "Indicadores Interseccionales y Medidas de Inclusión Social en Instituciones de Educación Superior" (págs. 14-20). MISEAL.

Cabero, J. y Ruiz-Palmero, J. (2017). Las Tecnologías de la Información y Comunicación para la inclusión: reformulando la brecha digital. International Journal of Educational Research and Innovation (IJERI), 9, 16-30. 
Caro, C. (2017). Posibilidades de acceso a la Universidad pública. Estudio interseccional con perspectiva de género sobre los perfiles de las personas aspirantes y admitidas a la Universidad Nacional de Colombia 2010-2017 [tesis de maestría, Universidad Nacional de Colombia].

https://repositorio.unal.edu.co/bitstream/handle/unal/60944/TESIS\%20Cindy\% 20Caro\%20C\%c3\%a1rdenas.pdf?sequence=1\&isAllowed=yo

Castaño, C. (2008). Nuevas tecnologías y género. La segunda brecha digital y las mujeres. Mujeres en Red.

Cecchini, S. (2005). Indicadores Sociales en América Latina y El Caribe. Estudios Estadísticos y Prospectivos. Cepal/ Naciones Unidas.

Chiroleu, A. (2014). Desigualdades en educación superior y políticas públicas. Los casos de Argentina, Brasil y Venezuela. Universidades, (59), 9-22.

Collins, P. y Bilge, S. (2016). Intersectionality. Polity Press.

Compaine, B. (2001). The Digital Divide: Facing a Crisis or Creating a Myth? MIT Press.

Connell, R. (1987). Gender and Power: Society, the person and Sexual Politics. Redwood City: Standford University Press.

Connell, R. (1995). Masculinities. Polity Press.

Connell, R. (2014). The Sociology of Gender in Southern Perspective. Current Sociology, 62(4), 550-567.

Cook, R. y Cusack, S. (2010). Estereotipos de género: perspectivas legales transnacionales. Bogotá. Pro-Familia.

Cooper, B. (2015). Intersectionality. En L. Disch y M. Hawkesworth, The Oxford Handbook of Feminist Theory (pp. 1-15). Oxford.

Coronado, S., Sandoval, S. y Torres, A. (2012). Relaciones interpersonales virtuales en los procesos de formación de investigadores en ambientes a distancia. Sinéctica, $39,1-14$.

D'Addio. (2007). Integenerational Transmission of Disadvantage: Mobility or inmobility cross Generations? A review of the evidence for OECD Countries. OECD Social, Employment and Migration Working Papers. 
Departamento Administartivo Nacional de Estadística. (1970). Boletín mensual de estadística. DANE.

Díaz, E. (2020). Liderazgo transformacional y equidad de género: el caso de estudiantes de posgrado. Universidad \& Empresa, 22(39), 1-21.

Eagly, A. \& Carli, L. (2012). Women and the labyrinth of leadership. Contemporary Issues in Leadership, 147-162.

Fernández, J. y Hernández, A. (2013). El liderazgo como criterio de calidad en la educación inclusiva. Estudios sobre Educación, (24), 83-102.

Foro Económico Mundial. (2006). Índice Global de la Brecha de Género. FEM.

Galtung, J. (2000). La violencia: cultural, estructural y directa. Cuadernos de Estrategia, $183,147-168$.

Gore, J. (1996). Pedagogías críticas y pedagogías feministas: cadversarias, aliadas u otra cosa? En Controversias entre las pedagogías: discursos críticos y feministas como regímenes de verdad (pp. 33-71). Morata.

Gurumurthy, A. (2004). Gender and icts. Institute of Development Studies, BRIDGE. http://ict4fisheries.org/wp-content/uploads/2016/12/GenderAndICTs.pdf

Herranz, C., Lorente, J. y Sánchez, I. (2017). Una mirada desde la interseccionalidad a la brecha digital. https://aecpa.es/files/view/pdf/congress-papers/13-0/1889/

López, I. (1992). El conocimiento sin exclusiones: la importancia de la revisión curricular. En Proyecto de Estudios de la Mujer. Hacia un currículo no sexista: integración de los estudios de la mujer y el género en los cursos introductorios de español, inglés y ciencias. Universidad de Puerto Rico.

Lopez, I. y Escalante, A. (2016). Análisis comparativo entre universidades española y mexicana. Sinéctica, (46), 1-20.

Lugones, M. (2005). Multiculturalismo radical y feminismos de mujeres de color. Revista Internacional de Filosofía Política, (25), 61-76. https://www.redalyc.org/pdf/592/59202503.pdf

Mancilla, M., Barros, D. y Mora, D. (2019). Identificación de brechas y perfil del género femenino en relación con su interacción con las tecnologías de la información. Revista Científica de la UCSA, 6(3), 63-73. 
Marcovici, K. (2002). El ungass, género y la vulnerabilidad de la mujer a la vih/sida en América Latina y el Caribe.

https://www.paho.org/Spanish/AD/GE/GenderandHIVSpanish.pdf

Martínez, J. (2020). La interseccionalidad como herramienta analítica para la praxis crítica del Trabajo Social. Reflexiones en torno a la soledad no deseada. Cuadernos de Trabajo Social, 33(2), 379-390.

Martínez, S. (2013). Desigualdad social y educación superior. Análisis sociológico comparado del caso de México. https://rua.ua.es/dspace/bitstream/10045/27539/1/Tesis_Martinez_Dordella.pdf

McCall, L. (2005). The Complexity of Intersectionality. Signs: Journal of Women in Culture and Society, 30(3), 1771-1800.

Moscovici, S. (1961). El psicoanálisis, su imagen y su público. Huemul.

Noticias ONU. (2020). Cierre de escuelas por el coronavirus: hay 850 millones de niños y jóvenes afectados.

https://news.un.org/es/story/2020/03/1470641\#: :text=El\%20cierre\%20de\%20e scuelas\%20en,brindando\%20apoyo\%20inmediato\%20a\%20los

Posada, L. (2017). Sobre Bourdieu, el habitus y la dominación masculina: tres apuntes. Revista de Filosofía, 73, 251-257.

Prieto, E. (2016). La necesidad de educar en comunicación digital en la sociedad global. Revista Linhas. Florianópolis, 17(33), 08-28.

Quintero, O. (2017). Aprendiendo la "R": Racialización y racismo prosaico en escuelas bogotanas. Revista Antropologías del Sur, 4(8), 105-125.

Rama, G. (1968). Origen social de la población universitaria. En G. Cataño, Educación y Sociedad en Colombia (p. 199). Universidad Pedagógica.

Romero, T. (2018). Cartilla Género. Ministerio de Justicia. https://minjusticia.gov.co/Portals/o/Conexi\%C3\%B3n/CajaHerramientas/genero Lartilla\%20G\%C3\%A9nero\%20final.pdf

Sáinz, M., Arroyo, L. y Castaño, C. (2020). Mujeres y digitalización. De las brechas a los algoritmos. Instituto de la Mujer y para la Igualdad de Oportunidades. 
Sherbin, L. y Rashid, R. (2017). Diversity doesn't stick without inclusion. Harvard Business Review Digital Articles.

Silvela, E. (2004). Manuel castells. la era de la información. individualismo y comunalismo en el origen de la violencia del siglo XXI. Cuadernos de Estrategia, 124, 147-172. https://dialnet.unirioja.es/descarga/articulo/2203829.pdf

Smith, E., \& Mackie, D. (1997). Psicología Social. Panamericana.

Social Watch. (2009). El índice de equidad de género social watch 2008. https://www.socialwatch.org/es/node/9939

Unesco. (1991). Informe mundial sobre la Educación. Unesco.

Unesco. (2016). Guía para la igualdad de género en las políticas y prácticas de la formación docente. https://unesdoc.unesco.org/ark:/48223/pfooo0260891_spa

Viveros, M. (2016). La interseccionalidad: una aproximación situada a la dominación. Debate Feminista, 52, 1-17. 